\title{
The use of liquid crystal panels for the study of holograms
}

\section{Iemmi, P. Cañizares, A. Moreno, J. Campos, M. Yzuel}

C. lemmi, P. Cañizares, A. Moreno, J. Campos, M. J. Yzuel, "The use of liquid crystal panels for the study of holograms," Proc. SPIE 9664, Ninth International Topical Meeting on Education and Training in Optics and Photonics, 96640Y (24 October 2005); doi: 10.1117/12.2207703

SDIE Event: Ninth International Topical Meeting on Education and Training in Optics and Photonics, 2005, Marseille, France 


\title{
Ref ETOP042
}

\section{The use of liquid crystal panels for the study of holograms}

\author{
C. lemmi ${ }^{1}$, P. Cañizares ${ }^{2}$, A. Moreno², J. Campos ${ }^{2}$, M. J. Yzuel ${ }^{2}$
}

${ }^{1}$ Departamento de Física, Facultad de Ciencias Exactas y Naturales, Universidad de Buenos Aires, (1428) Buenos Aires, Argentina

${ }^{2}$ Departamento de Física, Universidad Autónoma de Barcelona, 08193 Bellaterra, Spain

\section{Abstract}

This contribution corresponds to a laboratory work for a course in Holography and Optical Image Processing in a master degree. The spatial light modulators allow the display of computer generated holograms in real time. They permit the study of the features of image formation by holograms. We use a spatial light modulator made with a liquid crystal panel and polarization devices to get either amplitude or phase only responses. A convergent difractometer has been built to obtain the impulse response of the Fourier holograms. Different strategies to design the holograms have been proposed and experimental results are given.

spatial light modulators, holography, education

\section{Key words}

\section{Summary}

An optional course on Optical Image Processing and Holography exists in the Physics degree at the Autonomous University of Barcelona. The students are very much motivated by holography and its applications. It attracts young people in the field of optics and photonics. In this contribution, we propose the use of a liquid crystal panel to display computer generated holograms and to study their impulse responses.

The twisted nematic liquid crystal panels have been used in pattern recognition, image processing and holography ${ }^{1,2}$. This is mainly due to the low cost, their availability, and their capability to display images at video rates and to act as a programmable spatial light modulator (SLM).

Twisted-nematic liquid crystal panels are based in liquid crystal material in nematic phase. In this case, if there is no applied voltage, the molecules are oriented following a helical structure, but parallel to the sides of the panel. When an external voltage is applied, the molecules tend to get aligned along the electric field. This effect changes the polarization properties of the liquid crystal. This way, by using polarizers, a coupled phase and amplitude modulation is obtained ${ }^{3}$. An optimization of the modulation response is possible by adding linear polarizers and retarder plates to the TNLC panel ${ }^{4}$. This way, we built a spatial light modulator (LCSLM) that allowed us the possibility of obtaining either amplitude only response or phase only response.

In Figure 1 a sketch of the designed convergent difractometer is shown. We use an Ar laser beam working at $\lambda=458 \mathrm{~nm}$ as a light source. With a retarder plate $\lambda / 2$, laser polarization is rotated and coincides with the axis of polarizer $\mathrm{P} 1$ of the figure. A spatial filter expands the 
beam and the pinhole image is projected on the CCD plane by the convergent lens $L 1$. The laser beam impinges at a liquid crystal display Sony LCX012BL with VGA resolution $(640 \times 480$ pixels) extracted from a Sony video-projector. We presented a physical model for twisted nematic liquid crystal panels ${ }^{5}$ that predicts very accurately their behavior. Then, it was possible to design the configuration of the polarizing elements used with the liquid crystal panel to produce the desired modulation (pure phase or pure amplitude) through the use of elliptically polarized light ${ }^{4,6}$. The liquid crystal panels permits us send a computer generated Fourier hologram described by $f(x, y)$ and to get the impulse response $F(u, v)$ in the CCD plane of the difractometer (Figure 1).

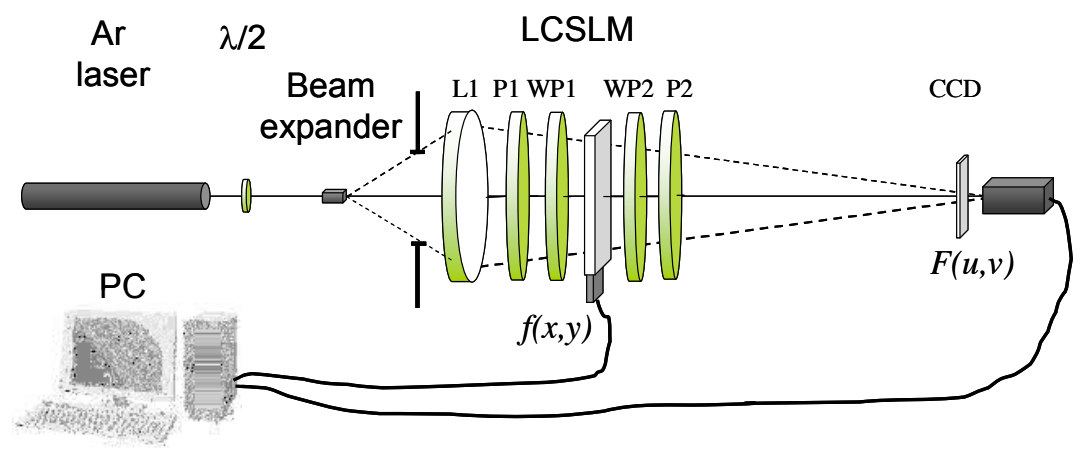

Figure 1. Convergent Difractometer. WP1 and WP2 are wave plates and P1 and P2 are polarizers. LCSLM is the liquid crystal spatial modulator.

The computer generated hologram that we use is a Fourier hologram that consists on the interference of the Fourier Transform of the scene and a reference plane wave tilted a known quantity. We use the LCSLM in phase only regime and we display the phase of the interference pattern. The impulse response is obtained with the CCD camera of the difractometer. The impulse response is shown in Figure 2(a). One can see that the scene (UAB) is reconstructed correctly and it is shifted with respect to the zero order diffraction. This effect is originated by the tilt of the plane wave front that interferes with the Fourier transform of the input scene. If there is not a tilt, the image obtained is centred in the zero order diffraction. The Figure 2(b) corresponds to the same case as in Figure 2(a) but a noised phase has been introduced in the input scene to make more uniform the power spectrum.

With this set-up the students can display different types of computer generated holograms and they can change dynamically parameters like the reference wave tilt. We can analyse the features of their impulse responses like the edge enhancement, position of the images, etc. Several strategies to improve the visualisation of the reconstructed scene are developed. 


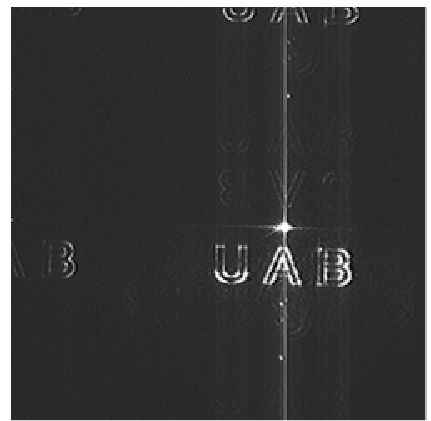

(a)

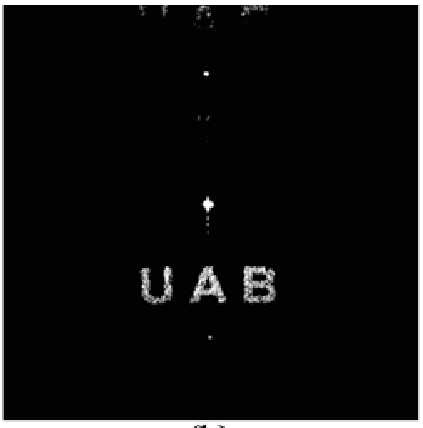

(b)

Figure 2. Reconstructed image when (a) the input scene has a null phase and (b) the input scene has a noised phase.

\section{Acknowledgements}

This work have been partially financed by the Ministerio de Ciencia y Tecnología under project BFM2003-006273-C02-01. C. lemmi acknowledge the support of CONICET and UBA of Argentina and Secretaría de Estado de Educación y Universidades of Spain.

\section{References}

[1] H.-K. Liu, J. A. Davis and R. A. Lilly, "Optical-data-processing properties of a liquid-crystal television spatial light modulator," Opt. Lett. 10, 635-637 (1985).

[2] H.-K. Liu and T.-H. Chao, "Liquid crystal television spatial light modulators," Appl. Opt. 28, 4772-4780 (1989).

[3] J. L. de Bougrenet de la Tocnaye and L. Dupont, "Complex amplitude modulation by use of liquid-crystal spatial light modulators," Appl. Opt. 36, 1730-1741 (1997).

[4] A. Marquez, C. lemmi, I. Moreno, J. A. Davis, J. Campos, M. J. Yzuel, "Quantitative prediction of the modulation behaviour of twisted nematic liquid crystal displays based on a simple physical model " Opt. Eng., 40, 2558, (2001)

[5] A. Márquez, J. Campos, A. Moreno, A. Robert, C. lemmi, J.A: Davis, M.J.Yzuel, "Characterization of edge in twisted nematic liquid crystal displays", Opt. Eng., 39, 3301-3307, (2000)

[6] J. Nicolás, J. Campos, M.J. Yzuel, "Phase and amplitude modulation of elliptic polarization states by non-absorbing anisotropic elements: application to liquid crystal devices", J. Opt. Soc. Am. A 19, pg.1013-1020 (2002). 